\title{
Le sol milieu vivant, un territoire qui reste à découvrir et à valoriser
}

\section{Soil as a living environment, a medium to be discovered and put to good use}

Oléagineux, Corps Gras, Lipides. Volume 7, Numéro 6, 490-3, Novembre - Décembre 2000, Dossier : "Agriculture, recherche et territoire"

Auteur(s) : Jean-Claude Cleyet-Marel, Philippe Hinsinger, UMR Symbioses tropicales et méditerranéennes, Inra/IRD/Cirad/Agro-M, Campus international de Baillarguet TA10/J, 34398 Montpellier Cedex 5, France.

Résumé : Le sol, théâtre de nombreuses transformations initiées par des organismes vivants, voit son fonctionnement perturbé par les pratiques de l'agriculture intensive. C'est le cas notamment du cycle de l'azote qui est fortement modifié par les apports massifs de fertilisants azotés de synthèse et ceci au détriment de la fixation biologique dont le potentiel est de moins en moins valorisé. Globalement, I'utilisation de fertilisants chimiques et de pesticides a conduit à minimiser et à négliger le potentiel biologique des sols. L'évaluation de la qualité des sols devra désormais intégrer la composante biotique et les mesures biologiques devront passer dans la pratique courante pour compléter les analyses physico-chimiques traditionnelles. Une meilleure valorisation du potentiel naturel des sols passera très certainement par une meilleure maîtrise des interactions dans la rhizosphère, lieu privilégié de rencontre entre la plante et les micro-organismes du sol.

Mots-clés : azote, fixation de l'azote, sol, qualité biologique

Summary : Soil functions which refers to diversity and activity of living organisms are often modified by modern high-intensity agriculture. Global nitrogen economy has been changed by synthetic nitrogen fertilisers use and biological nitrogen fixation is less and less valorised. The soil biological potential and its implication in global soil fertility are minimised by massive utilisation of chemical fertilisers and pesticides. In a next future soil quality couldn't be evaluated without an appreciation of biological properties. Conceptual and methodological approaches involved in soil biology will be useful tools in complement of traditional soil analysis. Management of the interactions in the rhizosphere which is a unique environment where plant root and soil microorganisms meet together is a way to better use of the soil biological potential.

Keywords : nitrogen, nitrogen fixation, soil, biological soil quality, organic matter, rhizosphere.

des sols, matière organique, rhizosphère. 


\section{ARTICLE}

Les préoccupations et les interrogations de la société civile et de la communauté scientifique en matière de qualité de vie et d'environnement conduisent les milieux agricoles à mettre en place de nouvelles stratégies de production et de gestion du patrimoine naturel [1]. Au-delà des débuts hésitants de l'agriculture " biologique " ou " raisonnée " et avec, à terme, l'instauration par les pouvoirs publics de redevances sur les excédents d'azote, c'est une gestion différente des assolements et des sols qui est attendue. Le niveau actuel de productivité agricole a été atteint en Europe de l'Ouest au prix de modifications importantes au niveau des cycles des éléments minéraux impliqués dans la nutrition des plantes. L'accès facile et peu onéreux aux fertilisants chimiques et notamment azotés ainsi que l'utilisation d'herbicides, de fongicides et d'insecticides ont conduit progressivement, au cours de la seconde moitié $d u x x^{e}$ siècle, à considérer le sol comme un simple support pour les végétaux et à oublier que c'est aussi et surtout un milieu vivant siège de multiples transformations et lieu d'intenses interactions biotiques, en particulier entre les racines et les microorganismes du sol. La diversité et la complexité des populations des êtres vivants présents dans le milieu tellurique, vers de terre, insectes et multiples autres invertébrés, nématodes, protozoaires, champignons, algues et bactéries ne présentent une richesse qui est encore très imparfaitement explorée et exploitée, au sens noble du terme. Cette complexité de l'écosystème tellurique est aussi une réalité qui a longtemps freiné l'acquisition des connaissances et rend encore malaisées les applications potentielles issues des travaux de recherche. Le sol n'est pas une ressource renouvelable, du moins à l'échelle humaine, et, derrière l'opposition entre une agriculture dite " conventionnelle " et une agriculture " organique ", c'est aussi la préservation de ce patrimoine qui est en cause.

\section{L'azote : un élément dont le cycle est fortement modifié par l'activité humaine}

Les modifications radicales apportées à la gestion de l'azote par les pratiques agricoles sont l'occasion d'illustrer les perturbations induites par les activités anthropiques sur les flux d'éléments dans le sol et, au-delà, sur les équilibres biologiques. Si l'azote n'est qu'un des éléments qui ont permis les immenses gains de productivité au cours des cinquante dernières années, il est un bon " marqueur " des pratiques culturales. II est à la fois, parmi tous les éléments impliqués dans les grands cycles biogéochimiques, celui qui affecte de la façon la plus significative la productivité végétale et celui dont le cycle est, en dehors des apports de fertilisants de synthèse, très largement contrôlé par des processus microbiens. La théorie des assolements développée au cours du xix siècle a été fondée essentiellement sur des acquis scientifiques reposant sur la chimie et les polémiques sur l'azote se sont déroulées pour l'essentiel à une époque où la microbiologie était une science qui restait à inventer. On savait néanmoins, sur la base d'observations rigoureuses, qu'un blé succédant à un trèfle ou à une luzerne bénéficiait d'une meilleure alimentation en azote et le slogan de l'époque était : "Veux-tu du blé ? Fais des prés » [2]. Cet adage se justifiait par le prix de revient des fertilisants et les quantités disponibles ne leur permettaient de jouer qu'un rôle d'appoint. Il était alors primordial de disposer de fertilisants organiques produits sur place et, pour cela, un certain effectif de bétail était nécessaire. Pour nourrir celui-ci, des superficies non négligeables devaient être consacrées aux fourrages. Cette pratique est liée au fait que, contrairement aux autres éléments minéraux majeurs entrant dans la composition des plantes, l'azote n'est pas issu, en milieu naturel, de l'altération de la roche-mère. 
La principale réserve d'azote est dans l'atmosphère, sous une forme non utilisable par les végétaux. Son entrée dans l'écosystème sol-plante est le résultat de l'activité de micro-organismes particuliers présents dans le sol (parmi ceux-ci, les rhizobium sont les plus connus). Ces derniers possèdent l'information génétique et la capacité de synthétiser le complexe enzymatique capable de réduire l'azote moléculaire $\left(\mathrm{N}_{2}\right)$ de l'atmosphère en ammonium. On estime généralement que plus de 100 millions de tonnes sont fixées annuellement dans le monde par les systèmes biologiques. Les systèmes les plus performants sont ceux qui associent la fixation biologique à la photosynthèse. La capacité de faire coïncider dans une même unité fonctionnelle la synthèse de composés carbonés et azotés à partir du gaz carbonique et de l'azote de l'air est un phénomène limité principalement, du moins pour les écosystèmes agricoles, aux légumineuses vivant en symbiose avec des bactéries fixatrices d'azote. On comprend ainsi l'intérêt de végétaux comme la luzerne ou le trèfle dans la pratique des assolements élaborée au xix siècle, usage qui est d'ailleurs resté en vigueur toute la première moitié $\mathrm{du} \mathrm{xx}^{\mathrm{e}}$ siècle. Une des particularités de l'entrée naturelle de l'azote dans l'écosystème sol-plante par voie biologique est d'associer de façon étroite le cycle de l'azote et celui du carbone. Ceci explique également que, pendant très longtemps, la fertilité du sol, en particulier sa composante azotée, ait été associée de façon intuitive puis raisonnée à la teneur en matières organiques du sol. Ce lien étroit et incontournable entre la teneur totale du sol en matières organiques et le pouvoir nutritif azoté du sol a été cassé avec la mise au point de la synthèse de l'ammoniac par le procédé Haber.

Au cours des quarante dernières années, les quantités de fertilisants azotés issus de la synthèse chimique utilisées dans le monde ont été multipliées par sept et, actuellement, plus de 70 millions de tonnes de fertilisants azotés sont épandues sur les terres agricoles. L'apport d'azote sous forme minérale a pour corollaire de dissocier les entrées de carbone et d'azote dans le sol et cette particularité n'est sans doute pas étrangère à la diminution assez systématique et continue du taux d'humus dans les sols agricoles.

Globalement, l'utilisation de fertilisants azotés est créatrice de biomasse, mais celle-ci est essentiellement exportée et les restitutions de composés organiques vers le sol sont bien souvent réduites au minimum. Par ailleurs, des techniques de préparation du sol pour le semis favorisant la minéralisation de la matière organique, le brûlage des pailles de céréales, le désherbage des vignobles sont autant de pratiques qui participent également à l'appauvrissement systématique des sols en matières organiques. La synthèse d'azote combiné à partir de l'azote atmosphérique en utilisant l'énergie fossile a conduit à négliger complètement l'entrée naturelle de l'azote dans les sols agricoles. Ainsi, les systèmes de culture actuels sont nettement orientés vers des rotations où les cultures fourragères n'ont plus ou peu de place. Les recherches sur la fixation biologique de l'azote ont du mal à trouver une finalité et des applications potentielles. Celles-ci sont pourtant nombreuses et variées. On peut à titre d'exemple, pour les milieux dégradés où le sol a été détruit et où les apports de fertilisants minéraux de synthèse ou organique sont inappropriés, envisager l'utilisation des plantes fixatrices d'azote pour réactiver le milieu. D'autres applications à caractère plus directement agricole sont l'enherbement contrôlé des vignes (photo) ou la production de protéines végétales, tâche pour laquelle les légumineuses et en particulier les fourragères comme le trèfle sont particulièrement efficaces [3]. Les événements récents qui ont ébranlé la filière de l'alimentation du bétail pourraient bien, en outre, relancer l'intérêt d'une augmentation de notre capacité de produire des protéines végétales à l'aide, en particulier, de légumineuses à graines comme le soja, mais aussi les lupins et les pois. L'utilisation de ce potentiel nécessite cependant d'importantes modifications 
des itinéraires techniques actuels. On peut espérer, dans le cadre d'une nouvelle politique agricole européenne orientée vers une désintensification et avec la mise en place des contrats territoriaux d'exploitation, voir une évolution vers des solutions techniques où sera plus judicieusement valorisé le potentiel biologique de l'entrée de l'azote dans l'écosystème sol-plante. À ce titre, bien que les écarts sur le plan des contraintes pédo-climatiques et socio-économiques soient considérables, le succès d'autres modèles de production tels que l'agriculture et l'élevage extensifs en Australie ou en Amérique du Sud devrait être source d'inspiration et d'espoir pour le futur. Ainsi, dans le modèle australien, les légumineuses à graines ou fourragères jouent un rôle central dans les rotations et assurent une entrée majeure d'azote dans l'écosystème cultivé. L'impossibilité économique de ces pays à intensifier à outrance, au même titre que ce qui a été accompli en France et en Europe occidentale, les a conduits sur des pistes de recherche assez différentes, cédant la place à une innovation forte, notamment en direction de la sélection d'espèces et de génotypes de plantes, mais aussi d'auxiliaires symbiotiques adaptés aux contraintes du milieu. Si jusqu'alors notre choix a été plutôt de trouver les moyens de lever ces contraintes à tout prix, il n'est pas trop tard pour reconsidérer d'autres options.

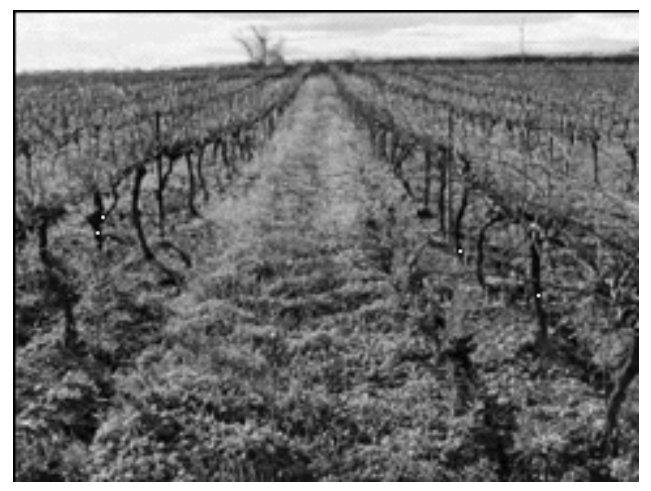

Photo. L'enherbement semi-permanent et contrôlé des vignes dans le Sud de la France, une alternative à l'utilisation massive d'herbicides. L'emploi d'une légumineuse annuelle (Trifolium subterraneum), dont les semences ont été bactérisées au moment du semis avec une souche de rhizobium, contribue au maintien et à l'enrichissement $d u$ sol en matière organique. Les cycles végétatifs décalés de la vigne et de la légumineuse limitent la compétition pour l'eau tout en assurant une bonne couverture du sol pendant la période hivernale à fortes précipitations, ce qui favorise l'infiltration de l'eau et limite l'érosion et les pertes de matières (fertilisants, pesticides, sol).

\section{La fraction organique, mémoire active du sol : vers une évaluation de la qualité biologique des sols}

La fertilité des sols est une notion imprécise dans sa définition et évolutive dans le temps. Ainsi, aux aspects technique et économique il convient aujourd'hui d'ajouter un volet environnement. Ce type de préoccupations environnementales nous amène précisément à nous questionner désormais sur ce qu'est la qualité des sols : maintenant qu'elle est de plus en plus menacée par de multiples activités anthropiques et nombre de pratiques agricoles, le besoin de savoir la définir et la mesurer apparaît d'autant plus grand. 
La qualité des sols est très largement appréciée à l'aide d'analyses physico-chimiques qui ont révélé elles-mêmes de multiples limites qui sont encore loin d'être levées, y compris pour des éléments majeurs tels que potassium et phosphore et plus encore pour les métaux (qu'il s'agisse d'oligoéléments nutritifs ou de métaux toxiques).

Cependant, dans le diagnostic de la qualité des sols, c'est incontestablement la composante biologique qui est encore trop négligée, voire absente. La gestion des besoins des plantes à l'aide de fertilisants minéraux, la relative simplicité d'emploi des méthodes de lutte chimique et leur efficacité à court terme ont fait passer au second plan le rôle fondamental de la fraction organique du sol, support de l'activité microbienne. Pourtant, les matières organiques sont à la base même de la lente élaboration du sol et elles sont un des éléments majeurs de sa fertilité, dans les trois dimensions que celle-ci comporte : physique, chimique et biologique. Si les végétaux ne se nourrissent pas à partir des molécules organiques du sol, la réorganisation permanente de la matière organique, suivie de sa minéralisation par biodégradation est source d'éléments minéraux, notamment de l'azote, du phosphore et du soufre. Globalement, les matières organiques jouent non seulement le rôle de réservoir d'éléments nutritifs indispensables à la croissance des plantes, mais participent aussi activement à la structuration du sol et, en améliorant sa résistance à la dégradation, limitent les effets de l'érosion.

La teneur en matières organiques d'un sol n'est pas constante, tant en quantité qu'en qualité, et varie considérablement selon le mode d'utilisation du sol. Il existe un lien étroit entre la teneur en carbone total du sol et la biomasse microbienne, de sorte que le statut organique des sols peut être estimé par la biomasse microbienne [4]. Cette dernière a un taux de renouvellement mille fois plus important que le carbone total et, en utilisant le rapport entre la biomasse microbienne et le carbone total, il est possible de mettre en évidence l'effet d'une modification de la gestion du sol sur une courte durée. L'évaluation des stocks de carbone dans des fractions granulométriques différentes du sol est également envisageable. Cette approche a notamment permis d'observer une bonne corrélation entre la biomasse microbienne et le carbone présent dans les fractions fines. Les mesures de respiration spécifique donnent également une idée de la taille du compartiment vivant du sol et de son taux de renouvellement apparent. Les déterminations d'activités enzymatiques sont aussi de plus en plus utilisées en raison de la facilité de leur mise en œuvre, mais l'interprétation des résultats reste souvent incertaine.

La microflore du sol, par sa diversité et son activité, est sans doute le maillon central de l'écosystème sol. On considère souvent le sol comme un immense réservoir d'espèces microbiennes mais le mode d'exploitation du sol modifie considérablement la biodiversité microbienne et, par voie de conséquence, le potentiel de transformation biologique du sol. On s'intéresse de plus en plus aux fonctions des matières organiques et de nombreuses applications sont attendues des travaux de recherche en cours. Par exemple, le solde entrées-sorties du bilan de l'azote, outil envisagé pour définir les pertes d'azote à l'échelle des exploitations agricoles, ne permet pas de hiérarchiser avec précision l'intérêt de différentes rotations au sein d'une même exploitation [5]. L'azote ne peut ainsi, de façon simpliste, être considéré dans sa globalité et il est nécessaire de considérer distinctement l'azote minéral et l'azote organique pour évaluer précisément les flux entre ces deux compartiments et la culture en place. On peut également, à terme, espérer évaluer les conséquences de pratiques culturales, d'itinéraires techniques, d'apports organiques ou de pesticides. 
Beaucoup d'efforts de recherche et de vulgarisation restent à effectuer pour rendre opérationnelles les mesures biologiques et les rendre complémentaires des analyses physico-chimiques classiques. Leur mise en œuvre reste lourde et la variabilité temporelle ou spatiale du sol rend l'interprétation des résultats encore délicate. Les travaux réalisés au cours des dix dernières années, notamment dans les sols viticoles, sont cependant riches de perspectives.

\section{La rhizosphère, un environnement particulier propice à la rencontre du végétal et de la microflore du sol}

La rhizosphère est un terme qui est apparu pour la première fois au début $d u x x^{e}$ siècle et qui caractérise la zone du sol entourant la racine. On distingue en général le rhizoplan qui est l'interface racine/sol et le sol rhizosphérique qui est situé au voisinage immédiat de la racine et soumis à son influence. La rhizosphère est donc un environnement particulier où les flux de matière et d'énergie entre le sol et la plante sont particulièrement intenses. La plante y mobilise l'eau et les éléments minéraux nécessaires à son développement et à sa croissance, induisant ainsi des modifications importantes du potentiel de l'eau et des concentrations ioniques du sol rhizosphérique. En outre, les échanges ioniques et gazeux entre les racines des végétaux et le sol contribuent à modifier de façon notable le $\mathrm{pH}$, le potentiel d'oxydo-réduction de la rhizosphère et, par suite, la biodisponibilité de nombreux éléments minéraux, nutritifs ou non [6]. Enfin et surtout, la racine libère dans la rhizosphère des quantités considérables de composés carbonés qui vont enrichir la fraction organique du sol. La rhizodéposition, terme désignant l'ensemble des composés organiques issus de la racine, est d'ailleurs un processus particulièrement actif et, pour reconstituer le stock organique d'un sol, la présence de prairies est plus efficace que l'enfouissement de résidus végétaux aériens. II est en outre estimé qu'un à deux tiers du carbone alloué par la plante à ses racines est exsudé et métabolisé par la microflore rhizosphérique, ce qui représente un flux de carbone correspondant à 10-50 \% de ce que la plante photosynthétise, suivant les espèces et les conditions environnementales [7].

De nombreux micro-organismes du sol sont des hétérotrophes pour le carbone et l'azote et toute une fraction de la microflore est stimulée au voisinage des racines des plantes, du fait de ce flux considérable de composés carbonés exsudés par la racine. Cet " effet rhizosphère " est désormais reconnu comme un processus majeur pour la vie microbienne du sol. L'enrichissement de la rhizosphère en micro-organismes est à la fois le résultat de leur multiplication et de leur mobilité propre qui leur permet de se déplacer et de se concentrer dans les zones riches en nutriments. Les modifications observées au niveau des populations de micro-organismes sont non pas seulement quantitatives mais également qualitatives car la plante exerce une sélectivité qui tend généralement à réduire la diversité microbienne et à favoriser des espèces ou des souches. Cette sélectivité est particulièrement poussée dans le cas de la symbiose fixatrice d'azote entre les légumineuses et les rhizobium où chaque légumineuse ne peut s'associer qu'avec un nombre réduit de souches bactériennes. La spécificité de cette reconnaissance est fondée sur l'échange de signaux moléculaires entre la plante et la bactérie. Des flavonoïdes particuliers excrétés par la racine sont reconnus par la bactérie qui, en réaction, synthétise des molécules complexes qui vont initier des réactions de la plante conduisant à la morphogenèse des nodosités, siège de la réduction de l'azote atmosphérique en ammonium. D'autres bactéries du sol, lorsqu'elles sont dans la rhizosphère, améliorent la croissance de ces dernières selon un mode d'action sans doute plurifactoriel. Certaines d'entre elles favorisent par exemple l'absorption de l'ion nitrate et la croissance de plantules de colza cultivées en 
conditions contrôlées [8]. Plusieurs souches bactériennes appartenant au genre Azospirillum sont employées dans le monde pour bactériser des cultures comme le maïs. Les champignons ectomycorhiziens ou endomycorhiziens sont également bien étudiés et sont utilisés en sylviculture.

L'activité des agents pathogènes des plantes est conditionnée non seulement par leur densité mais également par la densité et l'activité dans la rhizosphère de micro-organismes antagonistes. L'utilisation de fongicides systémiques, en supprimant les champignons endomycorhiziens, peut ainsi favoriser l'expression de pathogènes bactériens ou fongiques. D'une façon générale, il a été observé qu'une biomasse microbienne élevée et active limite la probabilité de rencontre entre un agent pathogène et la plante [9]. À ce mécanisme s'ajoutent des dispositifs de résistance plus particuliers liés à l'activité antagoniste de populations microbiennes spécifiques. II en va ainsi d'une maladie cryptogamique du blé, le piétin échaudage, qui est connue pour, paradoxalement, régresser au cours du temps lors d'une monoculture de blé (" assoupissement du piétin échaudage "), en raison de la sélection progressive d'une microflore antagoniste du champignon pathogène (Gaeumannomyces graminis var. tritici), en particulier de bactéries rhizosphériques du genre Pseudomonas [10].

D'une façon générale, une meilleure connaissance des équilibres microbiens dans la rhizosphère est devenue un enjeu majeur et, à terme, une meilleure gestion des interactions biotiques et abiotiques devrait permettre de limiter les intrants, qu'il s'agisse de fertilisants ou de pesticides. Le potentiel biologique d'un sol peut être loin de l'optimum et la plante est le meilleur moyen de modifier les équilibres microbiens. C'est le cas notamment avec les bactéries symbiotiques des légumineuses lorsqu'elles sont absentes ou présentes en nombre insuffisant dans le sol. La sélection et l'introduction de bactéries efficaces sur les semences au moment du semis permet ainsi, en Europe, à des légumineuses comme le soja ou la luzerne en sols acides de fonctionner avec l'azote de l'air. II a également été montré, aux États-Unis, qu'il était possible de trouver par sélection variétale des génotypes de soja capables de sélectionner une souche bactérienne particulière et, ainsi, de l'imposer lorsque d'autres souches moins efficaces appartenant au même groupe de spécificité sont présentes dans le sol. La plante elle-même, au travers de l'activité de ses racines, est capable de mettre en œuvre une batterie de moyens efficaces pour s'adapter aux contraintes chimiques, physiques et biotiques imposées par le sol. Dans notre agriculture productiviste, les schémas de sélection variétale sont très mal adaptés pour tirer parti des atouts dont disposent les végétaux et, en particulier, des interactions avec le cortège microbien rhizosphérique associé. En effet, ils reposent encore largement sur des sélections portant essentiellement sur une productivité maximale en conditions de sol non limitantes (irrigation et fertilisation pléthorique), c'est-à-dire dans des conditions de plus en plus éloignées de celles qu'une agriculture parcimonieuse au niveau des intrants se doit désormais de réaliser. II conviendrait donc de revenir à des schémas de sélection fondés sur d'autres critères tels que la rusticité, la résistance aux pathogènes et aux contraintes abiotiques (carences, toxicités, etc.) et, plus généralement, l'adaptation à produire en conditions suboptimales, c'est-à-dire en présence de contraintes avérées [11]. Alternativement, une autre façon de valoriser ces performances dont sont dotées certaines espèces ou génotypes consiste à repenser profondément les modes de conduite agronomiques : en innovant en matière de rotations (engrais verts fournissant azote, phosphore et matières organiques) et même d'associations (cultures associées, enherbement des vignes et vergers, agroforesterie, etc.), de façon à ce que les spécificités de chacune des espèces soient valorisées au mieux (notamment leur aptitude diverse à puiser dans les ressources nutritives et hydriques $d u$ sol). Cette innovation ne nécessite peut-être pas énormément d'imagination... mais un peu d'humilité pour réadapter des pratiques anciennes et les 
appliquer avec rigueur sur la base de connaissances scientifiques bien établies afin de gérer parcimonieusement notre capital sol milieu vivant. Dans ce même ordre d'idée, les pratiques développées actuellement dans le cadre de l'agriculture " biologique " gagneraient à être reconsidérées et (ré)évaluées dans la perspective de pouvoir, à terme, être transférées en dehors du strict cadre de cette agriculture, faisant de l'agriculture "biologique " un prototype d'agriculture différente, source d'innovation technique pour l'agriculture « conventionnelle » [12].

\section{REFERENCES}

1. TILMAN D (1998). The greening of the green revolution. Nature, $396: 211-2$.

2. SCORRAILLE G (1986). La fertilisation d'hier à aujourd'hui, connaissances et pratiques. Culture Technique, $16: 78-84$.

3. POCHON A (1981). La prairie permanente à base de trèfle blanc. Paris : Édition de I'Institut technique de l'élevage bovin ; $104 \mathrm{p}$.

4. CHAUSSOD R (1996). La qualité biologique des sols. Évaluation et implications. Étude et Gestion des Sols, $3: 261-78$.

5. REAU R, GIGANDON C (2000). Les indicateurs de pertes d'azote en systèmes de culture colzacéréale : cas du solde azote apport-export. Les Rencontres annuelles du Cetiom. Colza 2000, 30 novembre : 18-23.

6. HINSINGER P (1998). How do plant roots acquire mineral nutrients ? Chemical processes involved in the rhizosphere. Advances in Agronomy, 64 : 225-65.

7. LAMBERS H, SCHEURWATER I, ATKIN OK (1996). Respiratory patterns in roots in relation to their functioning. In : WAISEL Y, ESHEL A, KAFKAFI U, eds. Plant roots. The hidden half. 2nd Ed. New York: Marcel Dekker Inc. : 529-56.

8. BERTRAND H, PLASSARD C, PINOCHET X, TOURAINE B, NORMAND P, CLEYET-MAREL JC (2000). Stimulation of the ionic-transport system in Brassica napus by a plant growth-promoting rhizobacterium (Achromobacter sp.). Can J Microbiol, 46 : 229-36.

9. LEMANCEAU P, HEULIN T (1998). La rhizosphère. In : Sol : interface fragile. Paris : Inra : 93-106.

10. DAVET P (1996). Vie microbienne du sol et production végétale. Paris : Collection Mieux comprendre, Inra Éditions ; 383 p.

11. MEYNARD JM, DEBAECKE P, DEJOUX JF, SAULAS P (1997). Quelle sélection variétale pour une agriculture durable ? OCL, $4: 426-30$.

12. RIBA G, GAUTRONNEAU Y, BELLON S, SYLVANDER B, SAVINI I (2000). L'agriculture biologique et I'Inra - Vers un programme de recherche. Inra Mensuel. Tiré à part n ${ }^{\circ} 104$ mars-avril $2000 ; 25$ p. 